\title{
A Self-Organized One-Neuron Controller for Artificial Life on Wheels
}

\author{
Claudius Gros ${ }^{1}$, Laura Martin ${ }^{1}$ and Bulcsú Sándor ${ }^{1}$ \\ ${ }^{1}$ Institute for Theoretical Physics, Goethe University Frankfurt, Germany \\ gros07[at]itp.uni-frankfurt.de
}

\begin{abstract}
We study simulated animats in terms of wheeled robots with the most simple neural controller possible - a single neuron per actuator. The system is fully self-organized in the sense that the controlling neuron receives uniquely the actual angle of the wheel as an input. Non-trivial locomotion results in structured environments, with the robot determining autonomously the direction of movement (time-reversal symmetry is spontaneously broken). Our controller, which mimics the mechanism used to transmit power in steam locomotives, abstracts from the body plan of the animat, working without problems also in the presence of noise and for chains of individual two-wheeled cars. Being fully compliant our controller may be also used, in the spirit of morphological computation, as a basic unit for higher-level evolutionary algorithms.
\end{abstract}

\section{One neuron per joint controller}

Research in artificial life (Bedau et al., 2000) ranges from open ended evolution (Ruiz-Mirazo et al., 2004) to morphological computation (Müller and Hoffmann, 2017). Here we examine whether non-trivial locomotion may arise as self-organized attracting states in sensorimotor loops (Martin et al., 2016), i. e. in the combined phase space of neuronal controller and the dynamics of the body and of the environment (Sándor et al., 2015).

- We find that a single neuron per joint is sufficient for a self-organized controller which fully abstracts from terrain modularities.

- The one-neuron controller generates self-sustained navigation autonomously both in structured environments and for nonholonomic constraints. Structural knowledge of the dynamics of the robot, required otherwise (Das et al., 2006), is not needed.

- We propose that the here developed controller may simplify the control problem when higher-lever evolutionary optimization algorithms would address the actuators not directly, but indirectly via the respective controlling neuron.

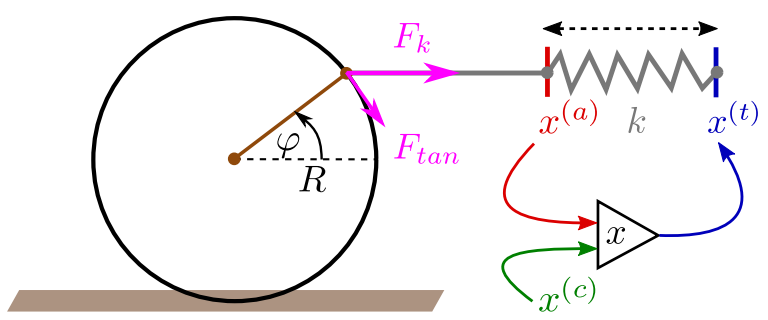

Figure 1: One neuron adaptive wheel controller. The membrane potential $x=x(t)$ of the single controlling neuron is determined by $\dot{x}=\Gamma\left(x^{(a)}-x\right)$, where $x^{(a)}=R \cos \varphi$ measures the current position of the wheel. The controller has hence access only to the angle $\varphi$ of wheel (propiosensation). The neural activity $y=y(x)=1 /(1+$ $\exp (-2 x))$ translates consecutively into a target position $x^{(t)}=2 y-1$ of a fictive transmission rod (in gray), which then couples elastically to the wheel via a spring with spring constant $k$. The torque $M=R F_{\text {tan }}$ results in the end from the force $F_{k}=k\left(x^{(t)}-x^{(a)}\right)$ exerted by the rod on the wheel, where $F_{\text {tan }}$ denotes the tangential component of $F_{k}$, and $R$ the radius of the wheel. An additional higher-order control signal $x^{(c)}$ would result, when added to the actual position $x^{(a)}$, in a compliant wheel.

\section{Simulated steam locomotive transmission}

Our controller mimics, as illustrated in Fig. 1, the power transmission mechanism used in steam locomotives, with the neural activity $y=y(t) \in[0,1]$ controlling to the position of a piston moving forth and back. The timescale $1 / \Gamma \approx(20-100) \mathrm{ms}$ induces a dynamic time-lag, which breaks the otherwise perfect $360^{\circ}$ symmetry of the setup. The direction of motion is determined consequently by the initial conditions, with both turning direction of the wheel being equally probable.

We fitted the two-wheel animats illustrated in Fig. 2 with our neural controller, with each of the two wheels controlled independently. The wheels are hence coupled only through the mechanics of the body of the animat and through the environmental feedback. The resulting control is capable 


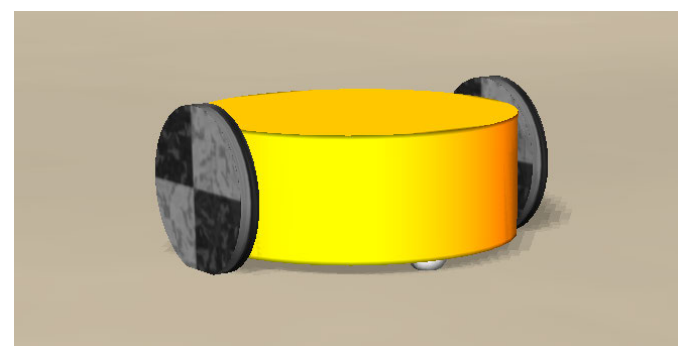

Figure 2: The simulated robot having two traction and two passive support wheels (black wheels on the sides, respectively small white balls below the body).

to deal adaptively both with holonomic and nonholonomic constraints (Fierro and Lewis, 1998).

We simulated the robots within the LPZRobots physics simulation environment (Der and Martius, 2012). The robot may not move at all, but this fixpoint is unstable, and small perturbation immediately induces a continuous acceleration, with the final velocity depending on the mass of the robot, the environmental friction, and the control parameters.

In Fig. 3 a sample path of the animat navigating autonomously in a complex environment is shown. Crossing from one inclined plane to another the robot first moves uphill, being however drawn downwards by gravitational forces - until it turns. It then speeds up again moving initially in half-circles transversely to the inclined plane.

Adding a higher level input control signal to the neuron, it is possible to modulate the navigation, fully retaining its dynamical flexibility thanks to its soft robotics architecture (Müller and Hoffmann, 2017). A regulation of the wheels rolling speed is achieved, to give an example, by constant control inputs. Compliant control is achieved here through the emergence of self-organized attractors in sensorimotorloop (Sándor et al., 2015).

We joined, in an additional experiment, the individual robots to trains of 5-7 cars, with friction applied only to the first car and with all 10-14 wheels being controlled independently (see Fig. 4). We find complex motion patterns that shows up as chaos both visually and in projection of the sensorimotor loop to the $\left(\varphi_{1}, \varphi_{2}\right)$ plane (Martin et al., 2016).

\section{References}

Bedau, M. A., McCaskill, J. S., Packard, N. H., Rasmussen, S., Adami, C., Green, D. G., Ikegami, T., Kaneko, K., and Ray, T. S. (2000). Open problems in artificial life. Artificial life, 6(4):363-376.

Das, T., Kar, I., and Chaudhury, S. (2006). Simple neuron-based adaptive controller for a nonholonomic mobile robot including actuator dynamics. Neurocomputing, 69(16):2140-2151.

Der, R. and Martius, G. (2012). The Playful Machine: Theoretical Foundation and Practical Realization of Self-Organizing Robots, volume 15. Springer Science \& Business Media.

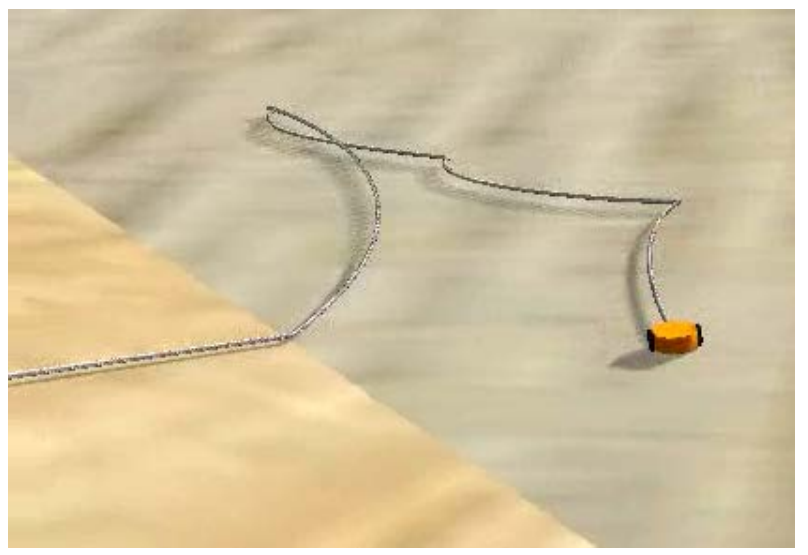

Figure 3: The simulated two-neuron two-wheel robot crosses from one inclined plane to another, with the silver line representing the past track. The observed motion is fully self-organized.

Fierro, R. and Lewis, F. L. (1998). Control of a nonholonomic mobile robot using neural networks. IEEE Transactions on neural networks, 9(4):589-600.

Martin, L., Sándor, B., and Gros, C. (2016). Closed-loop robots driven by short-term synaptic plasticity: Emergent explorative vs. limit-cycle locomotion. Frontiers in Neurorobotics, 10.

Müller, V. C. and Hoffmann, M. (2017). What is morphological computation? On how the body contributes to cognition and control. Artificial Life, 23:1-24.

Ruiz-Mirazo, K., Peretó, J., and Moreno, A. (2004). A universal definition of life: autonomy and open-ended evolution. Origins of Life and Evolution of Biospheres, 34(3):323-346.

Sándor, B., Jahn, T., Martin, L., and Gros, C. (2015). The sensorimotor loop as a dynamical system: How regular motion primitives may emerge from self-organized limit cycles. Front. Robot. AI, 2:31.

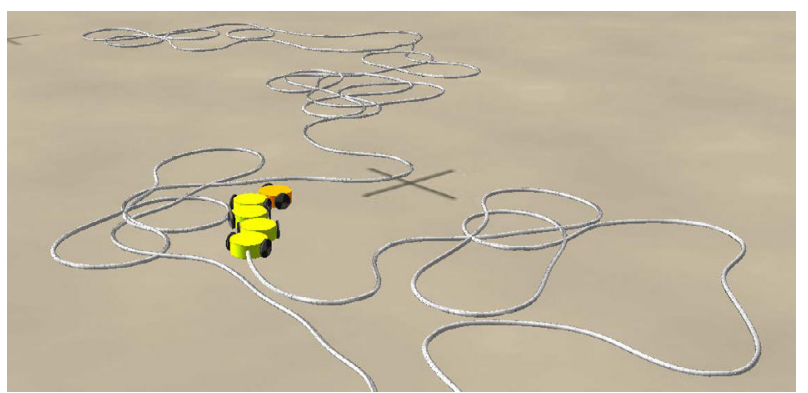

Figure 4: A train consisting of elastically coupled cars. The attractor resulting from a projection to a reduced phase space is manifestly not a limit cycle. 